\title{
The unfolded protein response is activated in Helicobacter-induced gastric carcinogenesis in a non-cell autonomous manner
}

\author{
Mhairi Baird ${ }^{1}$, Pei Woon Ang ${ }^{1}$, lan Clark ${ }^{2}$, Danial Bishop ${ }^{1}$, Masanobu Oshima ${ }^{3}$, Matthew C Cook ${ }^{4,5}$, \\ Christine Hemmings ${ }^{6}$, Shigeo Takeishi ${ }^{7}$, Dan Worthley ${ }^{7}$, Alex Boussioutas ${ }^{8}$, Timothy C Wang ${ }^{7}$ and Doug Taupin ${ }^{1}$
}

Mucous metaplasia (MM) is an aberrant secretory phenotype that arises during Helicobacter-induced gastric carcinogenesis. HSPA5, a key modulator of the unfolded protein response (UPR) activated by endoplasmic reticulum (ER) stress is overexpressed in gastric cancer (GC). We studied activation of the UPR in MM and GC in humans and mice. We assessed RNA and protein levels of ER stress markers (HSPA5, XBP1, and CHOP) in human GC, and correlated with Helicobacter pylori (H. pylori) status, then surveyed HSPA5 in normal gastric mucosa and gastric pre-neoplasia including gastritis and intestinal metaplasia (IM). The role of $H$. pylori infection in the UPR was assessed by co-culture with AGS GC cells. ER stress markers in metaplasia and dysplasia from transgenic K19-Wnt1/C2mE mice and C57BI/6 mice with chronic Helicobacter felis ( $H$. felis) infection were compared. HSPA5 was overexpressed in 24/73 (33\%) of human GC. Induction of HSPA5 and XBP1 splicing was associated with $H$. pylori-associated GC ( $P=0.007$ for XBP1 splicing). HSPA5 was overexpressed in MM but not gastritis in patients with $H$. pylori infection. Stimulation of AGS cells with CagA-positive H. pylori suppressed HSPA5 expression and XBP1 splicing. In the normal gastric mucosa of human and mouse, HSPA5 was constitutively expressed in MIST1-positive chief cells. Increased Hspa5 and Chop expression were found in dysplasia of C57BI/6 mice with chronic $H$. felis infection but was absent in spontaneous gastric dysplasia in $\mathrm{K} 19-\mathrm{Wnt} 1 / \mathrm{C} 2 \mathrm{mE}$ mice with concomitant loss of Mist 1 expression, similar to that observed in H. pylori-associated human GC. Induction of the UPR in the milieu of Helicobacter-induced chronic inflammation and MM may promote neoplastic transformation of Helicobacter-infected gastric mucosa.

Laboratory Investigation (2013) 93, 112-122; doi:10.1038/labinvest.2012.131; published online 29 October 2012

KEYWORDS: endoplasmic reticulum stress; germfree; mucous metaplasia; unfolded protein response

Helicobacter pylori (H. pylori) is a major risk factor for human gastric cancer (GC). ${ }^{1}$ Sustained interaction between H. pylori and the gastric epithelium induces chronic inflammation and loss of parietal cells (oxyntic atrophy), leading to development of mucous metaplasia (MM) - intestinal metaplasia (IM) and spasmolytic polypeptide expressing metaplasia (SPEM). ${ }^{2}$ Studies in human and murine models indicate that IM and SPEM are critical neoplastic precursors in the cascade of gastric carcinogenesis. ${ }^{3-6}$

IM is characterized by the presence of intestinal goblet cells with simultaneous expressions of TFF3 and $\mathrm{MUC2}^{7}$ while SPEM, named for its characteristic expression of spasmolytic polypeptide (TFF2), expresses MUC6 and resembles deep antral gland cells. ${ }^{5}$ Reported studies suggest that SPEM, derived from transdifferentiation of chief cells, ${ }^{8}$ gives rise to IM and ultimately dysplasia in the setting of oxyntic atrophy and chronic inflammation. ${ }^{9}$ Despite characterization of the sequence and origin of metaplastic mucous cell lineage, the mechanisms responsible for neoplastic transformation of the secretory cell lineage are not understood.

Cancer cells are under constant endoplasmic reticulum (ER) stress as hypoxia and nutrient deprivation affects protein glycosylation and production of ATP, resulting in accumulation of misfolded protein. ${ }^{10}$ Cancer cells adapt to this

\footnotetext{
${ }^{1}$ Cancer Research, The Canberra Hospital, Garran, ACT, Australia; ${ }^{2}$ Capital Pathology, Deakin, ACT, Australia; ${ }^{3}$ Division of Genetics, Cancer Research Institute, Kanazawa University, Kakuma-machi, Kanazawa, Japan; ${ }^{4}$ Immunology Research, The Canberra Hospital, Garran, ACT, Australia; ${ }^{5}$ Australian National University Medical School, Canberra, ACT, Australia; ${ }^{6} \mathrm{ACT}$ Pathology, The Canberra Hospital, Garran, ACT, Australia; ${ }^{7}$ Division of Digestive and Liver Diseases, Columbia University Medical Center, New York, NY, USA and ${ }^{8}$ Peter MacCallum Cancer Centre, East Melbourne, VIC, Australia

Correspondence: Dr D Taupin, Cancer Research, The Canberra Hospital, Yamba Drive, Garran, ACT 2605, Australia.

E-mail: Doug.Taupin@act.gov.au

Received 3 May 2012; revised 18 July 2012; accepted 19 July 2012
} 
homeostatic imbalance by activating the unfolded protein response (UPR) through ER-associated machineries. ${ }^{11}$ The UPR initiates protective signaling pathways that reduce RNA translation and enhance the degradation of misfolded proteins through upregulation of HSPA5, CHOP and XBP1 splicing. Activation of the UPR has been shown to promote tumor growth and reduce sensitivity to chemotherapeutic agents. ${ }^{10,12}$

In GC patients, activation of the UPR, as evidenced by the upregulation of HSPA5 has been observed in up to $47 \%$ of gastric carcinoma and is associated with increased metastasis and poor prognosis. ${ }^{13,14}$ As IM and SPEM cause expansion of secretory lineage cells and aberrant expression of trefoil factors and acidic mucins, we hypothesized that this increase in protein synthesis within this precancerous MM might also induce ER stress and contribute to carcinogenesis. In this study, we demonstrate that the UPR is activated non-cell autonomously in Helicobacter-induced metaplasia and dysplasia in both the human and mouse models of GC, highlighting a significant role of the UPR in mediating the progression of metaplasia to dysplasia under chronic Helicobacter infection.

\section{MATERIALS AND METHODS}

\section{Human Gastric Tumors and Non-Neoplastic Gastric Tissue}

Two independent cohorts of fresh frozen human GC specimens were obtained from The Canberra Hospital Tumor Bank $(n=11)$ and Peter MacCallum Cancer Center $(n=18)$. Additionally, 73 formalin-fixed, paraffin-embedded GC resection specimens were assembled onto a tissue microarray. Tissue sections of $4 \mu \mathrm{m}$ were used for immunohistochemical analysis, with each resection specimen represented by four array spots. Biopsy specimens of normal gastric mucosa $(n=16)$ and gastritis $(n=8)$ were obtained from patients referred for upper GI endoscopy, while fresh frozen gastric tumor and adjacent non-neoplastic gastric tissue were obtained from GC patients undergoing surgical resection. Additionally, twelve gastritis or IM endoscopic biopsies from subjects without the history of GC were obtained. Fresh tissues were snap frozen in liquid nitrogen, then stored at $-80{ }^{\circ} \mathrm{C}$ until use. Clinicopathological data for all primary gastric tumors and biopsy specimens are provided in Supplementary Material (Supplementary Table 1 and Table 2, respectively). RNA was extracted using the Qiagen RNeasy kit (QIAGEN GmbH, Germany) while protein was isolated from total cell lysate using the Novagen Phosphosafe lysis buffer (EMD Biosciences, St Louis, MO, USA). Informed consent was obtained from all patients and the project was approved by the Australian Capital Territory Health Human Research Ethics Committee.

\section{Assessment of $\boldsymbol{H}$. pylori Status in Patient Sera and Gastric Biopsy}

Frozen sera from GC patients and individuals undergoing endoscopy were collected before surgical procedures and assessed for $H$. pylori IgG antibody using ELISA
(BioMerieux) according to the manufacturer's instructions. The corresponding formalin-fixed, paraffin-embedded sections of non-tumor tissue were assessed by immunohistochemistry for $H$. pylori (Dako, Denmark).

\section{Gastric Tissues from Mouse Models of SPEM and Dysplasia}

Gastric tissues from 78-week Helicobacter felis (H. felis, ATCC strain 49179)-infected and age-matched control C57Bl/6 mice were obtained as described. ${ }^{15}$ Adult germfree $\mathrm{C} 57 \mathrm{Bl} / 6$ mice were obtained from the Bioservice Department of the Walter Eliza Hall Institute (Victoria, Australia) and maintained in germfree conditions until killed. Gastric tissues from K19-/C2mE and K19-Wnt1/C2mE compound transgenic mice, age 20 weeks, exhibiting SPEM and dysplasia, and age-matched control C57Bl/6 mice, were obtained as described. ${ }^{16}$ RNA extraction and fixation of tissue were performed as described above.

\section{Immunohistochemistry}

Expression of HSPA5, TFF1, TFF2, TFF3, CDH1, GRB7, HER2 and MIST1 was assessed immunohistochemically with heat-induced antigen retrieval. Antibodies and details of labeling are described further in Supplementary Material. Controls for HSPA5 staining are shown in Supplementary Figure 3.

\section{Quantitation of RNA and Protein Levels of ER Stress Markers HSPA5, spliced XBP1 and CHOP in GC Cell Line, Mouse and Human Tissues}

Increased expression of $H S P A 5, X B P 1$ spliced variant and $C H O P$ are indicators of ER stress. ${ }^{10}$ RNA levels of HSPA5, $X B P 1$ spliced $(X B P 1-\mathrm{s})$ and unspliced $(X B P 1-\mathrm{u})$ transcripts, and $C H O P$ were assessed in human GC cell line, human and mouse gastric tissues using quantitative real-time PCR (qPCR) (BioRad) as described previously. ${ }^{11,17}$ RNA levels were calculated using the comparative $\mathrm{Ct}$ method ${ }^{18}$ using $\beta$-actin as the reference. XBP1 splicing was also assessed by reversetranscriptase PCR (RT-PCR) amplification of complementary DNA obtained from total RNA to allow detection of $X B P 1$-s and XBP1-u transcripts in the human GC. ${ }^{11}$ HSPA5 protein expression was assessed by immunoblotting (Cell Signaling Technology).

Acute infection of $H$. pylori and treatment of thapsigargin (TG) in GC cell lines, ELISA measurement of IL-8, microarray gene expression data mining and statistical analysis are described in the Supplementary Material.

\section{RESULTS}

\section{HSPA5 Expression is Increased in Human GC}

The clinicopathological and molecular features of 73 primary gastric tumors in relation to HSPA5 protein expression are shown in Table 1. Strong expression of HSPA5 was observed in $24 / 73(33 \%)$ of cases. No significant association was observed between the examined clinicopathological or molecular parameters and HSPA5 expression (Table 1). 
Table 1 HSPA5 expression and clinicopathological and molecular characteristics of $\mathbf{7 3}$ gastric tumors

\begin{tabular}{|c|c|c|c|c|}
\hline \multirow{2}{*}{$\begin{array}{l}\text { Clinicopathological } \\
\text { features }\end{array}$} & \multirow{2}{*}{$\begin{array}{l}\text { All cases } \\
(n=73)\end{array}$} & \multicolumn{2}{|c|}{ HSPA5 expression } & $P$-value \\
\hline & & $\begin{array}{l}- \text { to }+ \\
(n=49)\end{array}$ & $\begin{array}{c}++ \text { to } \\
+++(n=24)\end{array}$ & \\
\hline
\end{tabular}

Lauren's classification

Intestinal

Diffuse

Mixed/not classifiable

37
22
14

$\begin{array}{ll}24 & 13 \\ 17 & 5 \\ 8 & 6\end{array}$

Lymph node metastasis

Absent

Present

Unclassified

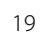

52

2

Tumor site

Antrum

Body

Cardia/gastroesophageal

junction

Unclassified

$\mathrm{CDH} 1$

Positive $(++$ to +++$)$

Negative $(-$ to + )

Unclassified

TFF1

Positive $(++$ to +++$)$
Negative $(-$ to +$)$
Unclassified

TFF2

Positive $(++$ to +++$)$
Negative $(-$ to +$)$
Unclassified

TFF3

$\begin{array}{lrrrr}\text { Positive }(++ \text { to }+++) & 15 & 10 & 5 & \\ \text { Negative }(- \text { to }+) & 50 & 31 & 19 & 0.23^{\mathrm{a}} \\ \text { Unclassified } & 8 & 8 & 0 & \end{array}$

aFisher's exact test was used when the sample size was $\leq 5$ to determine the statistical significance for comparison between each parameter and HSPA5 expression.

Over-Expression of HSPA5 is Associated with Constitutive Dysregulation of Other ER Stress Pathway Genes

Gene expression profile between normal gastric tissues and GC from the NCBI Gene Expression Omnibus revealed GC showed twofold or greater RNA expression of HSPA5 compared with non-tumor tissue (Supplementary Figure 1). ${ }^{19}$ Almost all of the eighteen genes showing concurrent dysregulation with HSPA5 in human cancers have putative functions in the ER stress-signaling pathway (Supplementary Table 3). The high enrichment of genes co-regulated with HSPA5 that are involved in ER stress-signaling suggests overexpression of HSPA5 in GC is a reflection of ER stress.

\section{Human GC with $H$. pylori Infection Demonstrates Increased HSPA5 Protein Expression and XBP1 Splicing}

ER stress activates the transmembrane sensor IRE1, an endoribonuclease (RNAse) that initiates a 26-nucleotide splicing of XBP1 mRNA. This spliced form of XBP1 mRNA acts as a transcription activator of ER chaperones, including HSPA5. ${ }^{11}$ Forty-five percent (13/29) of GC exhibited XBP1 splicing (Figure 1a). Of these, 92\% (12/13) were H. pyloripositive $(P=0.007)$. In addition eleven fresh GC specimens were assessed for HSPA5 protein expression. Elevated HSPA5 level was observed in 67\% (4/6) of $H$. pylori-positive GC, in contrast to increased HSPA5 expression in only one of five (20\%) H. pylori-negative GC (representative samples shown in Figure 1b). Two closely-spaced bands were observed on the immunoblot of HSPA5. The lower bands detected in the immunoblot of HSPA5 protein probably represent the alternative isoform of HSPA5va. ${ }^{20}$ Four of the five tumors exhibiting simultaneous XBP1 splicing and overexpression of HSPA5 were $H$. pylori-positive as verified by serological and immunohistochemical analyses (Supplementary Figure 2), while all six tumors (6/6) lacking both ER stress markers were H. pylori-negative. Of note, two patients (39 and 113) showed HSPA5 expression and splicing of $X B P 1$, respectively, in the non-neoplastic gastric mucosa (Figure 1a). We then assessed HSPA5, CHOP and XBP1 RNA levels in the endoscopic biopsy specimens of macroscopically and histologically normal gastric mucosa and gastritis. Despite considerable interindividual variation, there was no significant difference in RNA expression of the UPR markers in gastritis compared with normal gastric mucosa (Figure 1c).

\section{IM in Human is Associated with Increased HSPA5 Protein Expression Co-Localizing with Alcianophilia}

Taken together, the findings described raised the possibility that ER stress might be a pre-malignant response to H. pylori. We, therefore, assessed the presence of ER stress through immunohistochemistry of HSPA5 and its co-localization with acidic sialomucins in chronic gastritis and IM from GC patients with $H$. pylori infection. In the normal human stomach, low to scanty cytoplasmic expression of HSPA5 with occasional strongly positive cells was identified at the bases of gastric units (Figure 2a). In gastritis, where neutral mucins predominated, no upregulation of HSPA5 was observed (data not shown). In contrast, marked HSPA5 expression was evident in areas of IM and co-localized with alcianophilia 
a
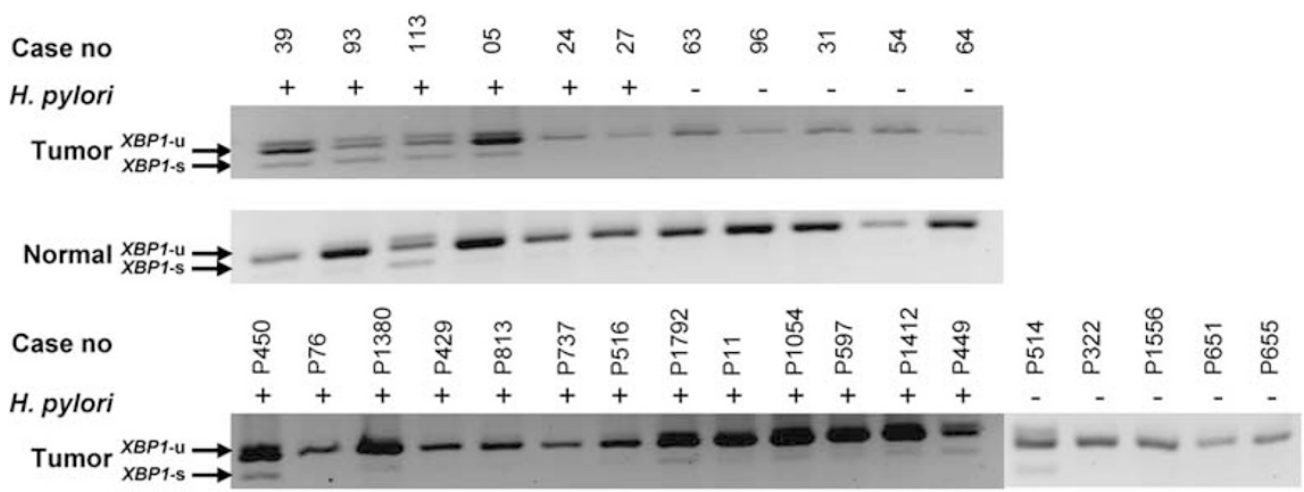

b
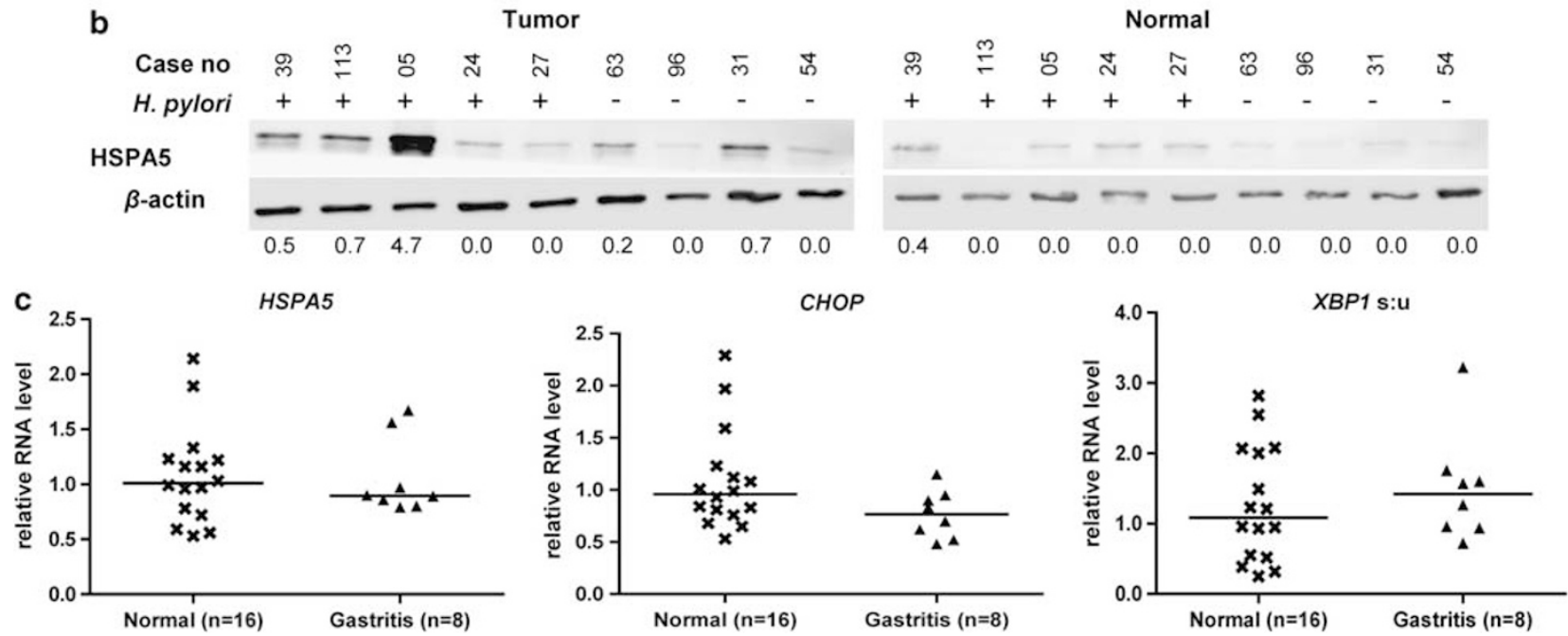

Figure 1 Endoplasmic reticulum stress is present in H. pylori-related human gastric cancer (GC). (a) XBP1 splicing in frozen gastric tumor and adjacent normal tissues when available. Reverse-transcriptase PCR showing the XBP1 unspliced (XBP1-u) and spliced (XBP1-s) transcripts differing by 26 bp. The $H$. pylori infection status of each GC patient is shown. Each amplicon was verified by sequencing. (b) Immunoblot of HSPA5 using $\beta$-actin as sample loading control. Mean level of HSPA5 quantified by densitometry from triplicate experiments using three sample lysates from each patient is indicated beneath the blot. (c) RNA levels of HSPA5, CHOP, XBP1-s and XBP1-u (expressed as XBP1-s:u ratio) relative to $\beta$-actin in normal gastric mucosa and gastritis as determined by quantitative real-time PCR. Each data point was normalized to the mean ratio of all normal gastric mucosa corrected to 1 . Horizontal line represents the median.

(Figure $2 \mathrm{~b}$ ). Increased expression of HSPA5 protein was also observed in GC although its distribution was independent of alcianophilia (top right corner of Figure 2c). These findings suggest that the presence of an aberrant secretory phenotype in MM might cause ER stress. However, as these samples also harbored GCs, we examined endoscopic biopsies in twelve individuals infected with $H$. pylori without GC to rule out a possible confounding field effect. Similar to the metaplastic lesion in GC patients, IM in patients without cancer also showed increased HSPA5 expression co-localizing with alcianophilia (Figure 2d). Upregulation of HSPA5 was also seen in the inflammatory infiltrates in these patients although no abnormality in HSPA5 expression in chronic gastritis was observed, as also seen by qPCR (Figure 1c). As recent studies have shown that transdifferentiation of chief cells contributes to gastric metaplasia in the human ${ }^{21}$ and mouse, ${ }^{8}$ we specifically examined HSPA5 expression in chief cells using MIST1 as a chief cell marker. In the normal human gastric mucosa, HSPA5 was expressed at a low level in the chief cell compartment at the bases of glands, correlating with MIST1 expression (Figure 2e). However, only occasional cells in this compartment were positive for both MIST1 and HSPA5 (Figure 2e, inset). This suggests that the chief cell is the site of HSPA5 expression in the normal stomach. We then examined HSPA5 and MIST1 expression in sections representing $H$. pylori-associated IM and dysplasia. There was a categorical increase in HSPA5 expression in IM and dysplasia, accompanied by complete loss of epithelial MIST1 expression (Figure 2f). MIST1 was instead noted in occasional inflammatory cells of the lamina propria in metaplasia (Figure $2 \mathrm{f}$, inset).

\section{Acute Infection of $\boldsymbol{H}$. pylori in GC Cell Line Does Not Induce ER Stress}

As activation of ER stress markers in GCs appeared to be related to $H$. pylori infection, we assessed whether acute 

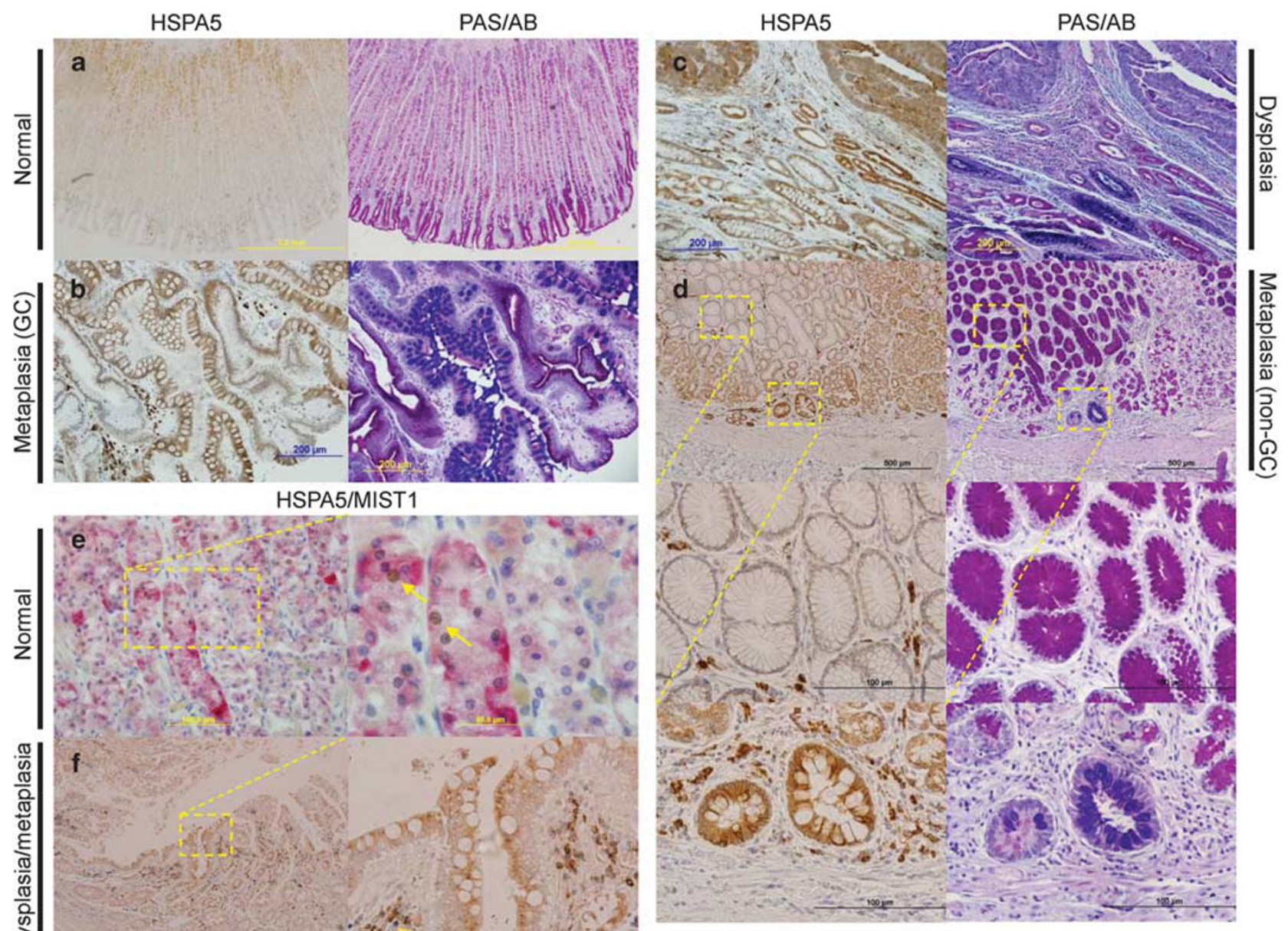

Figure 2 Increased HSPA5 in human gastric cancer (GC) and intestinal metaplasia (IM) is independent of MIST1 expression and colocalizes with alcianophilia in IM. (a) Normal human stomach showing low to scanty level of HSPA5 at bases of gastric units lacking alcianophilia. (b) IM in H. pyloripositive GC patient exhibits overexpression of HSPA5 co-localizing with alcianophilia. (c) The top right corner of the tissue exhibits areas of dysplasia with IM to the bottom left. In dysplasia, increased HSPA5 did not co-localize with alcianophilia. (d) Co-localization of increased HSPA5 with alcianophilia was evident in IM in H. pylori-positive non-GC patient. An area of AB-positive IM (bottom, inset) with upregulation of HSPA5 is bordered by areas of PAS-positive normal gastric glands (top, inset). (e) HSPA5 expression (red) localizes to chief cell compartment at the bottom of normal gastric glands with occasional cells staining positive for both MIST1 (brown) and HSPA5 (arrow). (f) In H. pylori-induced gastric metaplasia and dysplasia, increased HSPA5 (brown) is accompanied by loss of MIST1 (blue). Occasional plasma cells positive for MIST1 expression are identified in the lamina propria of the dysplastic region (arrow).

infection with H. pylori also induced ER stress. GC cell lines displayed variable activation of the UPR under basal conditions as evidenced by differential RNA levels of HSPA5, CHOP and XBP1-s (Figure 3a). Upon stimulation with TG, RNA expression of HSPA5, XBP1-s and $C H O P$ was markedly increased in all cell lines (Figure $3 b$ ) with decreased cell survival (Figure 3c). Although GC cell lines were able to mount the UPR under pharmacologically-induced ER stress, AGS cells co-cultured with a CagA-positive $H$. pylori strain suppressed HSPA5 protein expression (Figure 3d) and RNA levels of HSPA5, XBP1-s with non-significant increase in CHOP (Figure 3e). The level of HSPA5 expressed at $4 \mathrm{~h}$ postTG or $-H$. pylori treatment was similar to the level of untreated cells at $24 \mathrm{~h}$, likely reflecting the basal level of
HSPA5 constitutively synthesized in AGS cells under regular culture condition, as noted previously for most in vitro cultured cells.2 ${ }^{22}$ The discrepancy in protein (Figure 3d) and RNA levels of HSPA5 (Figure 3e) is consistent with a previous report of uncoupling of HSPA5 transcription and translation during ER stress. ${ }^{23}$ To confirm that this model of $H$. pylori infection was pathogenic, we confirmed an increase in IL-8 level in culture supernatant $24 \mathrm{~h}$ after bacterial challenge (Figure $3 \mathrm{f}$ ).

\section{ER Stress Arises in MM and Dysplasia Elicited By $H$. felis Infection in C57BI/6 Mice but not in Transgenic K19-Wnt1/C2mE and K19-C2mE Mice}

As the previous data demonstrate that $H$. pylori-associated IM and GC in human are associated with ER stress, we next 

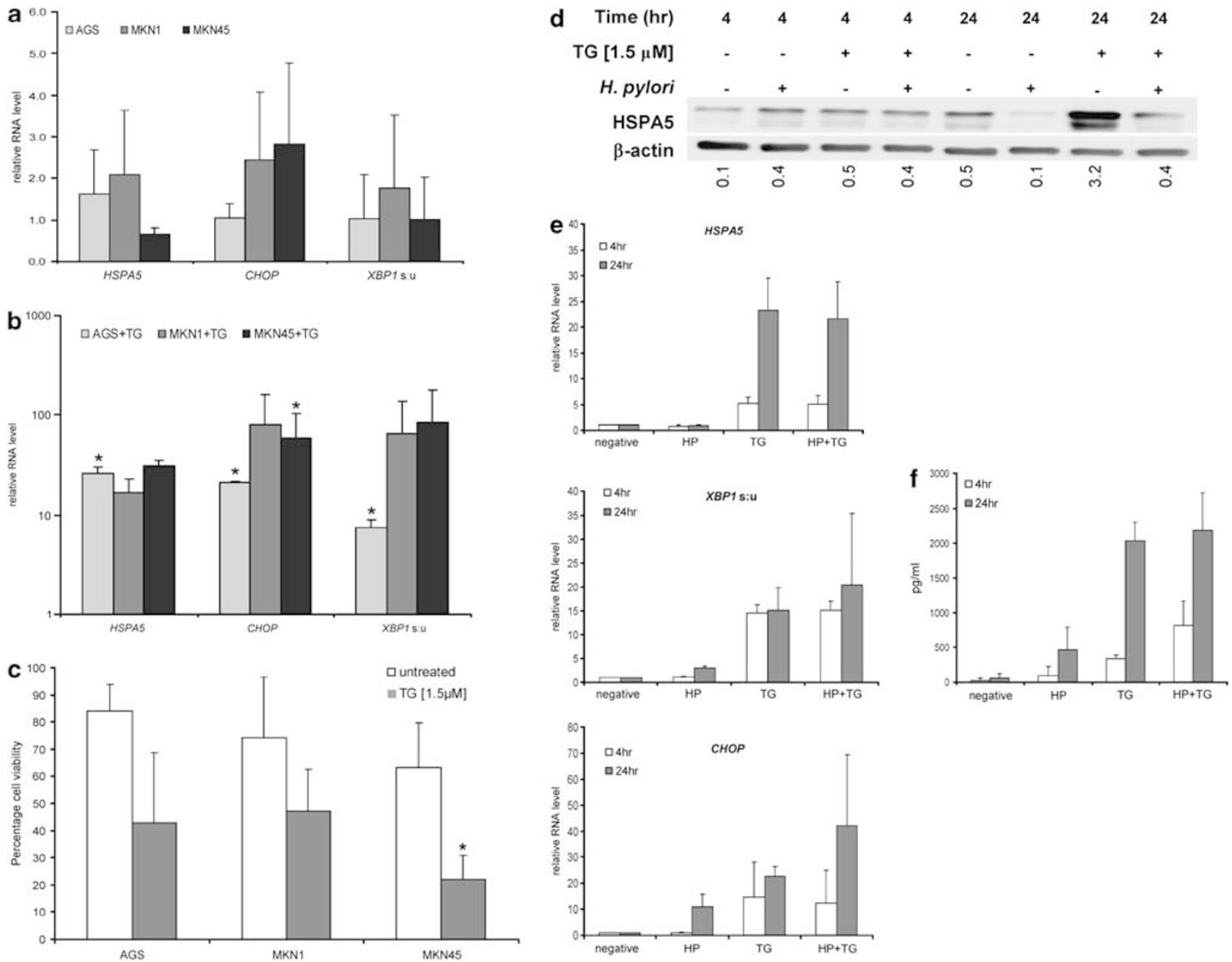

Figure 3 Differential activation of the unfolded protein response in gastric cancer (GC) cell lines and lack of endoplasmic reticulum stress during transient infection of AGS cells with CagA-positive $H$. pylori. (a) Relative RNA levels of HSPA5, CHOP and XBP1 (expressed as XBP1-s:u ratio) in GC cell lines under basal condition using AGS, chosen arbitrarily, as the calibrator. (b) Relative RNA levels of HSPA5, CHOP and XBP1 in GC cell lines treated with $1.5 \mu \mathrm{mol} / \mathrm{I}$ TG for $24 \mathrm{~h}$ compared with the corresponding cell line under basal condition. (c) Cellular proliferation as measured by MTT assay in cells treated with TG as described in (b). (d) HSPA5 protein expression in AGS cells co-cultured with $1.5 \mu \mathrm{mol} / \mathrm{l}$ TG or CagA-positive $H$. pylori for 4-24 h. Quantitation of HSPA5 expression by densitometry from triplicate experiments is indicated beneath the blot. (e) Relative RNA value of HSPA5, CHOP and XBP1 in AGS cells subjected to the same treatment as described in (d), normalized to $\beta$-actin, using untreated AGS cells as the calibrator. (f) Mean IL-8 induction in the AGS cells by CagA-positive $H$. pylori at 4 and $24 \mathrm{~h}$ post bacterial challenge. All data are represented as mean \pm s.d. from triplicate experiments. ${ }^{*} P \leq 0.02$ compared with untreated cells.

evaluated whether ER stress is induced in two independent mouse models of MM and dysplasia elicited by (i) H. felis infection in $\mathrm{C} 57 \mathrm{Bl} / 6$ mice and (ii) in transgenic K19-Wnt1/ $C 2 m E$ and $K 19-C 2 m E \mathrm{C} 57 \mathrm{Bl} / 6$ mice. Chronic infection of C57Bl/6 mice with $H$. felis causes atrophy, SPEM and dysplasia after 12-18 months, closely capturing the human GC cascade. $^{24}$ In this model, the metaplastic epithelium is frequently derived from inflammatory cells of bone-marrow origin, ${ }^{24}$ the development of gastric epithelial cancer is, therefore, non-cell autonomous. By comparison, the K19-Wnt1/ $C 2 m E$ and $K 19-C 2 m E$ transgenic mice (also on a $\mathrm{C} 57 \mathrm{Bl} / 6$ background) develop spontaneous MM and dysplastic gastric tumors by 20 weeks of age due to cell-intrinsic constitutive expression of the Wnt and prostaglandin $\mathrm{E}_{2}$ pathways. ${ }^{16}$ Wild-type C57Bl/6 mice infected for 78 weeks with $H$. felis exhibited abundant Hspa5 expression in the alcianophilic metaplastic region (Figure $4 \mathrm{a}$, right), similar to that seen in the human (Figure 2b). Increased Hspa5 expression was observed on the apical surface of dysplastic cells but did not co-localize with alcianophilia (Figure 4a, left). High Hspa5 expression was also found in the inflammatory cells of the dysplastic region (Figure $4 \mathrm{a}$, inset). In contrast, in the K19$C 2 m E$ and K19-Wnt1/C2mE mice with dysplasia, minimal Hspa5 was detectable in the epithelial cells and scattered 

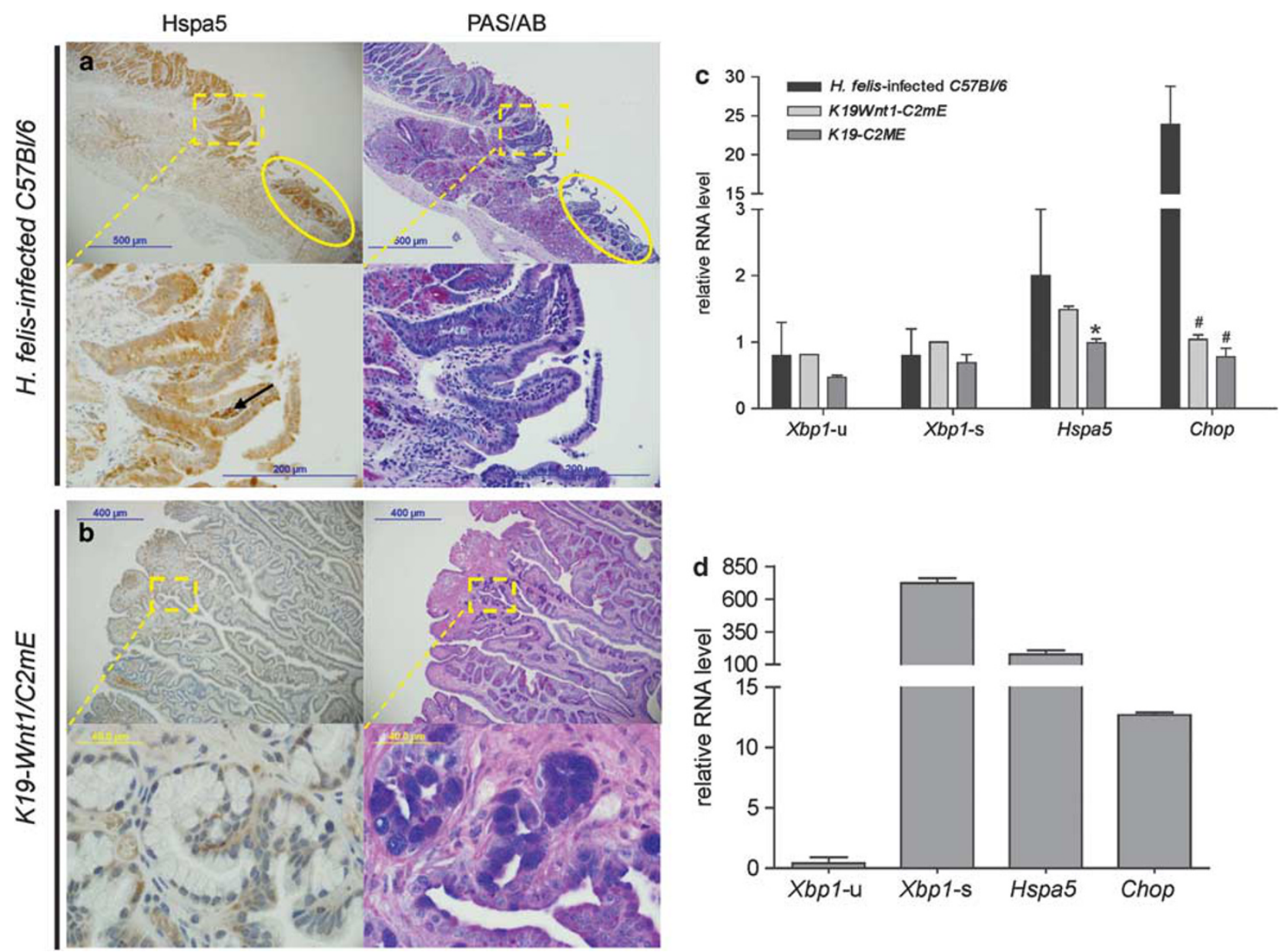

Figure $4 \mathrm{Hspa} 5$ is absent in the dysplasia of the K19-wnt1/C2mE mice but is upregulated in the dysplasia of $H$. felis-infected mice and colocalizes with alcianophilia in spasmolytic polypeptide expressing metaplasia (SPEM). (a) Increased Hspa5 expression (left) within SPEM (area enclosed in circle) and dysplasia (area enclosed in square) induced by 78-week infection of C57BI/6 mice with H. felis. Co-localization of Hspa5 with alcianophilia in SPEM is depicted by PAS/AB staining of serial section. (a, inset) Increased Hspa5 expression (left panel) on the apical surface of epithelial cells in dysplasia and in the inflammatory infiltrates (arrow). PAS/AB staining of serial section showing lack of Hspa5 co-localization with alcianophilia in the dysplastic region (right). (b) Minimal Hspa5 is expressed in the mucus metaplasia and dysplasia of the K19-wnt1/C2ME mice. Similar results were obtained for the K19C2mE mice (data not shown). PAS/AB staining of the corresponding section shows lack of alcianophilia in the metaplastic and dysplastic region.

(b, inset) Higher magnification of the same section (area enclosed in square) depicting low level of $\mathrm{Hspa} 5$ in the dysplastic and metaplastic cells and alcianophilic goblet cells. (c) Quantitative real-time PCR (qPCR) measurement of Xbp1-u, Xbp1-s, Hspa5 and Chop mRNAs in the H. felis-infected C57BI/6 mice, uninfected $K 19-W n t 1 / C 2 m E$ and $K 19 / C 2 m E$ mice. RNA levels are expressed as fold expression normalized to $\beta$-actin, relative to uninfected wildtype $\mathrm{C} 57 \mathrm{BI} / 6$ mice. ${ }^{*} \mathrm{Hspa} 5$ level was significantly increased in the $H$. felis-infected mice compared with the K19-wnt1/C2ME mice $(P=0.04)$. ${ }^{\#} \mathrm{Chop}$ was significantly higher in the $H$. felis-infected mice compared with the K19-Wnt1/C2ME mice $(P=0.02)$ or K19-C2ME mice $(P=0.01)$. (d) Average qPCR measurement of Xbp1-u, Xbp 1-s, Hspa5 and Chop mRNAs in NIH/3T3 cells treated with $1.5 \mu \mathrm{mol} / \mathrm{I}$ TG for $4 \mathrm{~h}$ relative to untreated cells. All data are represented as mean \pm s.e. from triplicate experiments.

Figure 5 Increased Hspa5 in Mist1-positive chief cells colocalizes with alcianophilia at the base of gastric glands in specific pathogen-free (SPF) and germfree (GF) C57BI/6 mice and transgenic K19-Wnt1/C2ME mice. (a) Uninfected wild-type SPF C57BI/6 mice showed marked Hspa5 expression at the base of gastric glands. The base of each gastric gland stains weakly for acidic sialomucins with $A B$ at $\mathrm{pH} 2.5$ while mucous neck cells and mucous pit cells stain for neutral mucins with PAS (upper). Non-neoplastic gastric mucosa of the K19-Wnt1/C2ME mice and GF C57BI/6 mice also express Hspa5 at the base of the gastric glands co-localizing with alcianophilia (middle and bottom). (b) Immunoblot of Hspa5 in SPF and GF C57BI/6 mice using $\beta$-actin as sample loading control. Mean Hspa5 level as measured by densitometry from triplicate experiments is indicated beneath the blot. (c) Relative RNA expression of Xbp1, Hspa5 and Chop mRNA in GF C57BI/6 mice normalized to $\beta$-actin, using wild-type SPF C57BI/6 mice as the calibrator. All data are represented as mean \pm s.e. from triplicate experiments. (d) Dual-staining of Hspa5 (brown) and Mist1 (blue) in the normal gastric mucosa of wild-type $\mathrm{C} 57 \mathrm{Bl} / 6$ and $\mathrm{K} 19-W n t 1 / \mathrm{C} 2 \mathrm{mE}$ mice showing expression of Hspa5 identified predominantly in Mist1-positive chief cells (upper and middle). Increased expression of $\mathrm{Hspa} 5$ throughout the gastric glands in $\mathrm{H}$. felis-induced dysplasia in wild-type $\mathrm{C} 57 \mathrm{BI} / 6$ mice was independent of loss of Mist 1 expression (lower). Occasional plasma cells showing positivity for Mist1 staining are found in the lamina propria (arrow). 

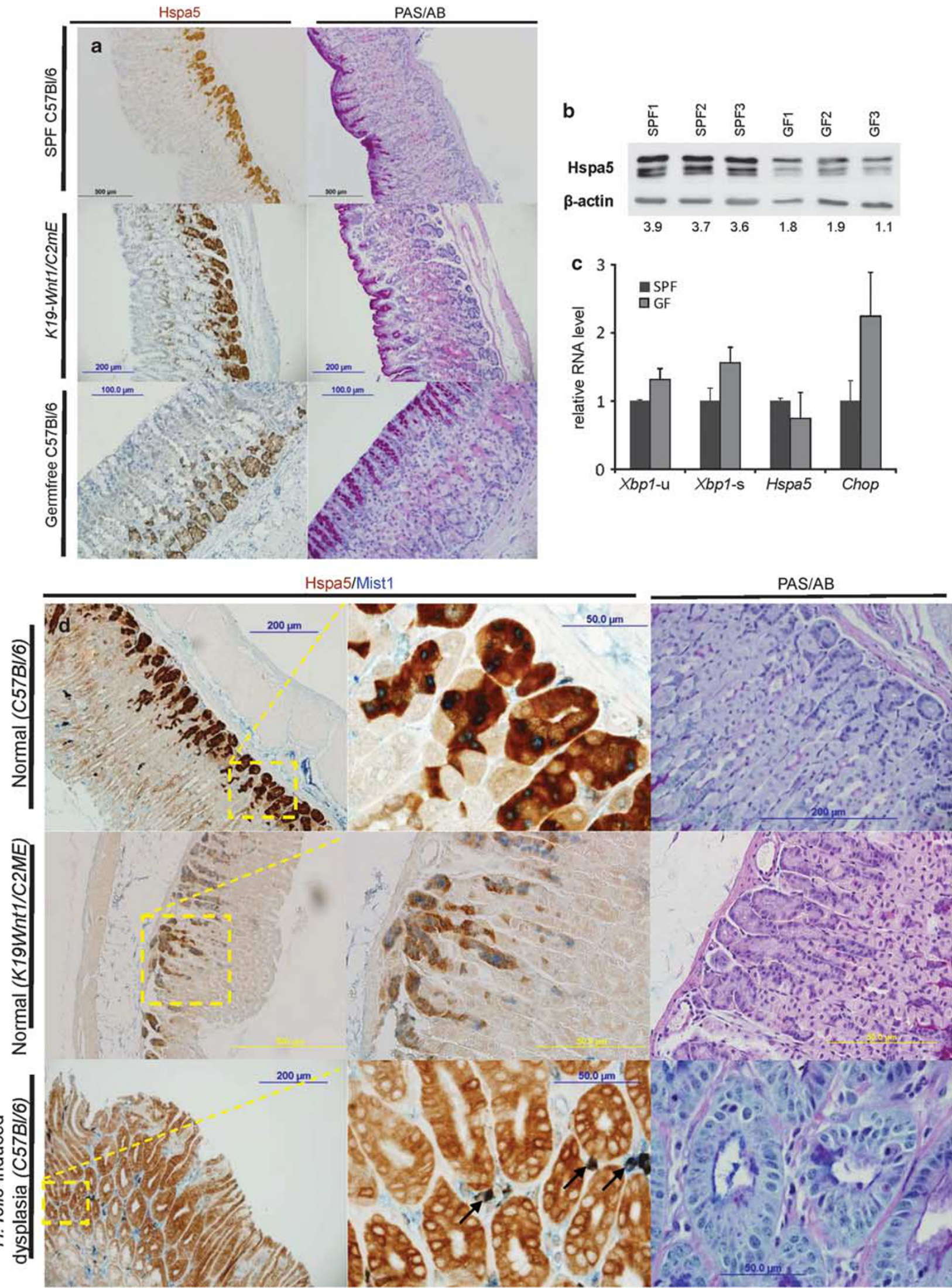
inflammatory cells (data from the K19-Wnt1/C2mE mice are shown) (Figure 4b). Expression of Hspa5, where present, did not co-localize with alcianophilia (Figure 4b). We then compared Hspa5 RNA levels in dysplasia from the two mouse models. Hspa5 RNA expression was significantly higher in the $H$. felis-infected $\mathrm{C} 57 \mathrm{Bl} / 6$ mice than the K19$W n t 1 / C 2 m E$ mice $(P=0.04)$ (Figure 4c). However, the quantitative difference in Hspa5 expression between $H$. felisinfected and K19-Wnt1/C2mE mice was relatively modest ( $\leq 2$-fold), while the RNA levels of Xbp1-s and Xbp1-u were not significantly different among the three groups (Figure 4c). By comparison, NIH/3T3 mouse fibroblast cells treated with $1.5 \mu \mathrm{mol} / 1 \mathrm{TG}$ for $4 \mathrm{~h}$ demonstrated a 700 -fold induction of spliced XBP1 and 150-fold induction of Hspa5 compared with untreated cells (Figure 4d). We speculated that the relatively modest increase in RNA levels of Hspa5 and Xbp1-s was due to the high basal level of expression of $\mathrm{Hspa} 5$ (and $\mathrm{Xbp1}$ ) in the normal mouse gastric gland (Figure 5c). The transcription factor Chop is induced by ER stress and is a marker of the pro-apoptotic arm of the UPR. $^{25}$ RNA expression of Chop was substantially and significantly higher in gastric mucosa of chronic $\mathrm{H}$. felisinfected $\mathrm{C} 57 \mathrm{Bl} / 6$ mice than in K19-Wnt1/C2mE mice $(P=0.02)$ and $K 19 / C 2 m E$ mice $(P=0.01)$ (Figure $4 c)$. The 25 -fold elevation of Chop RNA was comparable to the 12-fold induction of Chop in NIH/3T3 mouse fibroblast cells treated with TG compared with untreated cells (Figure 4d). Therefore, the H. felis model of GC but not the K19-Wnt1/ $C 2 m E$ model was associated with sustained induction of the UPR.

\section{Hspa5 is Expressed Predominantly in Chief Cells at the Base of Gastric Glands in Mice}

We observed that considerable Hspa5 expression could be detected at the base of the gastric glands in age-matched wild-type and transgenic mice with or without $H$. felis infection (Figure 5c). Qualitatively, gastric Hspa5 expression appears higher in the normal mouse than human stomach. This region of the gastric gland also stained positively with $\mathrm{AB}$ at $\mathrm{pH} 2.5$, consistent with previous reports in rodents but not in humans. ${ }^{26}$ An intact UPR is required for normal innate ${ }^{27,28}$ and adaptive immunity in metazoans and activation of innate immunity induces the UPR. ${ }^{29}$ High constitutive expression of $\mathrm{Hspa} 5$ in the normal gastric mucosa might, therefore, be associated with the indigenous gastric microflora of conventionally raised coprophagic mice. We examined ER stress markers in age-matched, germfree $\mathrm{C} 57 \mathrm{Bl} / 6$ mice and found that germfree $\mathrm{C} 57 \mathrm{Bl} / 6$ mice showed lower expression of $\mathrm{Hspa} 5$ protein than the specific pathogen-free (SPF) mice (Figure 5b), co-localizing with alcianophilia at the base of the gastric glands (Figure 5a). Despite this, the RNA levels of Hspa5 and other ER stress marker including Xbp1 and Chop did not show significant differences between the SPF and germfree $\mathrm{C} 57 \mathrm{Bl} / 6$ mice (Figure 5c).
As expression of Hspa5 was confined to the bases of murine fundic glands where chief cells normally reside, we determined the identity of Hspa5-expressing cells by double-labeling chief cells with Mist1. Hspa5 was expressed predominantly in Mist1-positive chief cells at the bases of fundic gastric units in histologically normal gastric mucosa in both the wild-type and K19-Wnt1/C2mE or K19-C2mE transgenic mouse models (Figure $5 \mathrm{~d}$, top \& middle). We observed that Mist1 expression was absent from the epithelia of MM and dysplasia in both the mouse models (Figure 5c, bottom), as seen in human IM and dysplasia (Figure 2f). However, in $H$. felis-induced dysplasia in wild-type C57BL/6 mice, marked epithelial Hspa5 expression was observed (Figure 5c, bottom). Furthermore, occasional plasma cells with intense MIST1 staining were identified in the lamina propria of the dysplastic region in C57BL/6 wild-type mice with $\mathrm{H}$. felis infection, as seen in human IM and dysplasia (Figure 2f) and consistent with a previous report. $^{8}$

\section{DISCUSSION}

This study demonstrates for the first time that the UPR is involved in the pathogenesis of Helicobacter-induced gastric tumorigenesis. Elevated expression of HSPA5 is constitutive with dysregulation of multiple UPR effectors and is associated with non-cell autonomous induction of ER stress in the Helicobacter-related cascade of neoplastic transformation in both the human and mouse gastric mucosa.

We found that HSPA5 was overexpressed in $33 \%$ of GC, consistent with previous reports. ${ }^{13,14}$ We observed that activation of the UPR was significantly associated with H. pylori-positive GC (12/13, 92\%). Increased expression of HSPA5 in the IM of $H$. pylori-positive subjects with and without GC rules out field cancerization and suggests induction of the UPR occurs early in the evolution of the metaplasia to dysplasia. Expression of HSPA5 coincident with the appearance of acidic mucins (alcianophilia) in the secretory cells of IM suggests that it could be aberrant expression of MUC2 protein that triggers the UPR. This observation is supported by findings of Heazlewood et $a l^{17}$ demonstrating induction of the UPR by misfolded MUC2 protein. As UPR activation was not observed in gastritis or transient infection of GC epithelial cell line as reported previously by Namba et al, ${ }^{30}$ we conclude that inflammation induced by $H$. pylori is necessary but insufficient to induce ER stress in the stomach.

Marked expression of Hspa5 co-localizing with alcianophilia was evident at the base of the gastric glands in the normal gastric mucosa of all mouse models independent of Helicobacter infection. Hspa5 is known to regulate differentiation of several cell types, ${ }^{31}$ and further evaluation of gastric cell type markers will be required to define the cell lineage origin of Hspa5-expressing metaplastic cells in the mouse. Focal expression of Hspa5 in mouse gastric mucosa is partly attributable to the natural microflora found in SPF 
mice as lower level of $\mathrm{Hspa} 5$ protein was detected in germfree mice. Accordingly, it is possible that under prolonged ER stress, innate immune response to commensal bacteria may promote proliferation of tumor cells through activation of the UPR.

Similar to the observations in human IM and GC, in $H$. felis-infected $\mathrm{C} 57 \mathrm{Bl} / 6$ mice, increased Hspa 5 was observed in metaplasia (co-localizing with alcianophilia) and dysplasia, in contrast to the lack of Hspa5 expression in SPEM and dysplasia from the K19-Wnt1/C2mE and K19-C2mE mice. The contrasting expression of $\mathrm{Hspa} 5$ in the two independent mouse models of metaplasia and dysplasia supports the concept that ER stress does not arise in the absence of Helicobacter infection and chronic inflammation and is thus non-cell autonomous. During transition of metaplasia to Helicobacter-induced dysplasia, increased expression of HSPA5 along the entire gastric glands in both the human and mouse models was accompanied by loss of MIST1, a marker for chief cells. This suggests that the UPR may have a mechanistic role in the transdifferentiation of mature chief cells into metaplasia as reported previously. 8,21

Lack of ER stress response during acute Helicobacter infection suggests activation of the UPR may be a reparative response of gastric epithelium to chronic Helicobacter infection. We hypothesize that in the Helicobacter-infected gastric mucosa, prolonged ER stress following chronic inflammation, compounded by aberrant mucin synthesis in the metaplastic cells triggers UPR activation. Cell death mediated by CHOP could promote development of oxyntic atrophy while proliferation driven by HSPA5 overexpression may trigger neoplastic progression within metaplasia. Induction of the UPR, therefore, creates a permissive state, wherein deleterious molecular changes are tolerated to allow malignant transformation of metaplastic cells. Once the cells have undergone neoplastic changes, subversion of the UPR by tumor cells to maintain ongoing tumourigenesis is independent of metaplasia-related ER stress as reflected by the lack of co-localization of HSPA5 with alcianophilia in the dysplastic cells. The UPR, as an adaptive response cells use to survive ER stress during chronic inflammation, may, therefore, drive metaplastic cells along the metaplasiadysplasia pathway.

Although we have provided evidence for the involvement of ER stress in $H$. pylori-related gastric carcinogenesis, there are several limitations in the current study. Owing to a limited quantity of GC tissue, we were unable to assess the RNA levels of all UPR markers in $H$. pylori-infected gastric tumors. Analysis of possible association between the overexpression of HSPA 5 and $H$. pylori infection status within the 73 GC was precluded as this information was not available. Further mechanistic studies in models of chronic ER stress are needed to corroborate potential causal links between induction of the UPR and Helicobacter-induced MM and gastric carcinogenesis. In conclusion, UPR activation in Helicobacter-associated gastric lesions may represent a primary, oncogenic event through which Helicobacter-bacteria induce GC in humans and mice in a non-cell autonomous fashion.

Supplementary Information accompanies the paper on the Laboratory Investigation website (http://www.laboratoryinvestigation.org)

\section{ACKNOWLEDGEMENTS}

This work was supported by grants from the National Health and Medical Research Council (DT), the Canberra Hospital Radiation Oncology Trust Fund (DT), the Canberra Region Medical Foundation (DT), and ESA International (DT).

Author contributions: MB, PWA and DB acquired and analyzed the results. IC, MO, MC, CH, ST, DW, AB, TCW, DT analyzed data and contributed critical clinical perspective to the results. MO developed K19-C2mE/Wnt1, K19C2mE mice. PWA and DT wrote the manuscript. DT designed the experiments and critically reviewed the manuscript. All authors have read and approved the final version of the manuscript. MB and PWA contributed equally to this work.

\section{DISCLOSURE/CONFLICT OF INTEREST}

The authors declare no conflict of interest.

1. Polk DB, Peek Jr RM. Helicobacter pylori: gastric cancer and beyond. Nat Rev Cancer 2010;10:403-414.

2. Correa P, Houghton J. Carcinogenesis of Helicobacter pylori. Gastroenterology 2007;133:659-672.

3. Filipe Ml, Munoz N, Matko I, et al. Intestinal metaplasia types and the risk of gastric cancer: a cohort study in Slovenia. Int J Cancer 1994;57:324-329.

4. Halldorsdottir AM, Sigurdardottrir M, Jonasson JG, et al. Spasmolytic polypeptide-expressing metaplasia (SPEM) associated with gastric cancer in Iceland. Dig Dis Sci 2003;48:431-441.

5. Weis VG, Goldenring JR. Current understanding of SPEM and its standing in the preneoplastic process. Gastric Cancer 2009;12: 189-197.

6. Yamaguchi $H$, Goldenring JR, Kaminishi $M$, et al. Identification of spasmolytic polypeptide expressing metaplasia (SPEM) in remnant gastric cancer and surveillance postgastrectomy biopsies. Dig Dis Sci 2002;47:573-578.

7. Goldenring JR, Nomura S. Differentiation of the gastric mucosa III. Animal models of oxyntic atrophy and metaplasia. Am J Physiol Gastrointest Liver Physiol 2006;291:G999-1004.

8. Nam KT, Lee HJ, Sousa JF, et al. Mature chief cells are cryptic progenitors for metaplasia in the stomach. Gastroenterology 2010; 139:2028-2037.

9. Goldenring JR, Nam KT, Wang TC, et al. Spasmolytic polypeptideexpressing metaplasia and intestinal metaplasia: time for reevaluation of metaplasias and the origins of gastric cancer. Gastroenterology 2010;138:2210 e1.

10. Ma $Y$, Hendershot LM. The role of the unfolded protein response in tumour development: friend or foe?. Nat Rev Cancer 2004;4:966-977.

11. Lin $\mathrm{JH}$, Li H, Yasumura $\mathrm{D}$, et al. IRE1 signaling affects cell fate during the unfolded protein response. Science 2007;318:944-949.

12. Dong $\mathrm{D}$, Ko $\mathrm{B}$, Baumeister $\mathrm{P}$, et al. Vascular targeting and antiangiogenesis agents induce drug resistance effector GRP78 within the tumor microenvironment. Cancer Res 2005;65:5785-5791.

13. Zhang J, Jiang Y, Jia Z, et al. Association of elevated GRP78 expression with increased lymph node metastasis and poor prognosis in patients with gastric cancer. Clin Exp Metastasis 2006;23:401-410.

14. Zheng HC, Takahashi $\mathrm{H}$, Li XH, et al. Overexpression of GRP78 and GRP94 are markers for aggressive behavior and poor prognosis in gastric carcinomas. Hum Pathol 2008;39:1042-1049.

15. Kurt-Jones EA, Cao L, Sandor F, et al. Trefoil family factor 2 is expressed in murine gastric and immune cells and controls both gastrointestinal inflammation and systemic immune responses. Infect Immun 2007;75:471-480. 
16. Oshima $\mathrm{H}$, Matsunaga A, Fujimura $\mathrm{T}$, et al. Carcinogenesis in mouse stomach by simultaneous activation of the Wnt signaling and prostaglandin E2 pathway. Gastroenterology 2006;131:1086-1095.

17. Heazlewood CK, Cook MC, Eri R, et al. Aberrant mucin assembly in mice causes endoplasmic reticulum stress and spontaneous inflammation resembling ulcerative colitis. PLoS Med 2008;5:e54.

18. Schmittgen TD, Livak KJ. Analyzing real-time PCR data by the comparative C(T) method. Nat Protoc 2008;3:1101-1108.

19. Hippo $\mathrm{Y}$, Taniguchi $\mathrm{H}$, Tsutsumi $\mathrm{S}$, et al. Global gene expression analysis of gastric cancer by oligonucleotide microarrays. Cancer Res 2002;62:233-240.

20. Ni M, Zhou H, Wey S, et al. Regulation of PERK signaling and leukemic cell survival by a novel cytosolic isoform of the UPR regulator GRP78/ BiP. PLos One 2009;4:e6868.

21. Lennerz JK, Kim SH, Oates EL, et al. The transcription factor MIST1 is a novel human gastric chief cell marker whose expression is lost in metaplasia, dysplasia, and carcinoma. Am J Pathol 2010;177: 1514-1533.

22. Li J, Lee AS. Stress induction of GRP78/BiP and its role in cancer. Curr Mol Med 2006:6:45-54

23. Gulow K, Bienert D, Haas IG. BiP is feed-back regulated by control of protein translation efficiency. J Cell Sci 2002;115:2443-2452.
24. Houghton J, Stoicov C, Nomura S, et al. Gastric cancer originating from bone marrow-derived cells. Science 2004;306:1568-1571.

25. Oyadomari S, Mori M. Roles of CHOP/GADD153 in endoplasmic reticulum stress. Cell Death Differ 2004;11:381-389.

26. Schumacher $U$, Duku $M$, Katoh $M$, et al. Histochemical similarities of mucins produced by Brunner's glands and pyloric glands: A comparative study. Anat Rec A Discov Mol Cell Evol Biol 2004;278:540-550.

27. Iwakoshi $\mathrm{NN}$, Lee $\mathrm{AH}$, Vallabhajosyula $\mathrm{P}$, et al. Plasma cell differentiation and the unfolded protein response intersect at the transcription factor XBP-1. Nat Immunol 2003:4:321-329.

28. Richardson CE, Kooistra T, Kim DH. An essential role for XBP-1 in host protection against immune activation in C. elegans. Nature 2010; 463:1092-1095.

29. Kaser A, Blumberg RS. Survive and innate immune response through XBP1. Cell Res 2010;20:506-507.

30. Namba T, Hoshino T, Suemasu $S$, et al. Suppression of expression of endoplasmic reticulum chaperones by Helicobacter pylori and its role in exacerbation of NSAID-induced gastric lesions. J Biol Chem 2010;285:37302-37313.

31. Sugiura K, Muro Y, Futamura K, et al. The unfolded protein response is activated in differentiating epidermal keratinocytes. J Invest Dermatol 2009;129:2126-2135. 